\title{
Estimating the magnetic moment of microscopic magnetic sources from their magnetic field distribution in a layer of nitrogen-vacancy (NV) centres in diamond
}

\author{
Janis Smits ${ }^{1}$, Andris Berzins ${ }^{1}$, Florian H. Gahbauer ${ }^{1, a}$, Ruvin Ferber ${ }^{1}$, Kaspars Erglis ${ }^{2}$, Andrejs Cebers ${ }^{2}$ \\ and Juris Prikulis ${ }^{3}$ \\ ${ }^{1}$ Laser Centre, University of Latvia, 19 Rainis Boulevard, 1586 Riga, Latvia \\ 2 Laboratory of Magnetic Soft Materials, University of Latvia, 19 Rainis Boulevard, 1586 Riga, Latvia \\ ${ }^{3}$ Institute of Chemical Physics, University of Latvia, 19 Rainis Boulevard, 1586 Riga, Latvia
}

Received: 31 August 2015 / Received in final form: 24 December 2015 / Accepted: 2 February 2016 Published online: 29 February 2016 - (c) The Author(s) 2016

\begin{abstract}
We have used a synthetic diamond with a layer of nitrogen-vacancy (NV) centres to image the magnetic field distributions of magnetic particles on the surface of the diamond. Magnetic field distributions of $4 \mu \mathrm{m}$ and $2 \mu \mathrm{m}$ ferromagnetic and $500 \mathrm{~nm}$ diameter superparamagnetic particles were obtained by measuring the position of the optically detected magnetic resonance peak in the fluorescence emitted by the NV centres for each pixel. We fitted the results to a model in order to determine the magnetic moment of the particles from the magnetic field image and compared the results to the measured magnetic moment of the particles. The best-fit magnetic moment differed from the value expected based on measurements by a vibrating sample magnetometer, which implies that further work is necessary to understand the details of magnetic field measurements on the micro scale. However, the measurements of two different types of ferromagnetic particle gave internally consistent results.
\end{abstract}

\section{Introduction}

The nitrogen-vacancy (NV) centre [1] shows great promise for magnetic field measurements $[2,3]$ (see the reviews by Rondin et al. [4] and Schirhagl et al. [5] for more information). Recently, NV centres ${ }^{1}$ have been used to image magnetic field distributions in physical $[6,7]$ and biological $[8,9]$ systems. Nevertheless, the measurements are not always easy to interpret, as it is difficult to control all parameters on a microscopic scale. For example, magnetic moments measured for magnetosomes inside magnetotactic bacteria differ by almost an order of magnitude depending on the method used $[10,11]$. In order to understand this promising technique better, it is helpful to apply it to particles of known magnetic moment, which will lead to optimised and more accurate measurement protocols.

Magnetometry using NV centres is made possible by the properties of the energy level structure of the NV centre in diamond, shown in Figure 1. NV centres can be excited into the phonon band above the ${ }^{3} E$ state by radiation with a wavelength in the green part of the

\footnotetext{
a e-mail: florian.gahbauer@lu.lv

1 The NV centres of interest here are $\mathrm{NV}^{-}$centres as opposed to $\mathrm{NV}^{0}$ centres, but we will not write the ${ }^{-}$henceforth.
}

spectrum, usually $532 \mathrm{~nm}$. Most of the fluorescence is emitted between wavelengths of $650 \mathrm{~nm}$ and $800 \mathrm{~nm}$ at room temperatures, with the zero phonon line at $637 \mathrm{~nm}$. The relative strengths of the nonradiative transitions by way of the singlet states allow the ground state with spin component $m_{S}=0$ to be preferentially populated when the NV centre is continuously excited, while reducing the fluorescence of an NV population excited with $m_{S}= \pm 1$. As a result, the spin state can be determined from the fluorescence intensity. The ground state exhibits a zero-field splitting of $2.87 \mathrm{GHz}$. The energies $E$ of the $m_{S}= \pm 1$ ground-state sublevels are changed by a magnetic field according to $\Delta E=\mu_{\mathrm{B}} g_{S} m_{S} B_{z}$, where $\mu_{\mathrm{B}}$ is the Bohr magneton, $g_{S}$ is the Landé factor for spin, $m_{S}$ is the $z$-component of the NV centre's spin and $B_{z}$ is the value of the magnetic field projected onto the NV centre axis. Thus, transitions can be induced between the ground state sublevels by a microwave field when its frequency is in resonance with the energy shift between the $m_{S}=0$ and $m_{S}= \pm 1$ components, and in the event of such a resonance, the overall fluorescence intensity decreases. The result is a so-called optically detected magnetic resonance (ODMR) spectrum. Two examples are shown in Figure 2. In the first, the field is parallel to one NV axis, which means that its projection along the other three axes is 


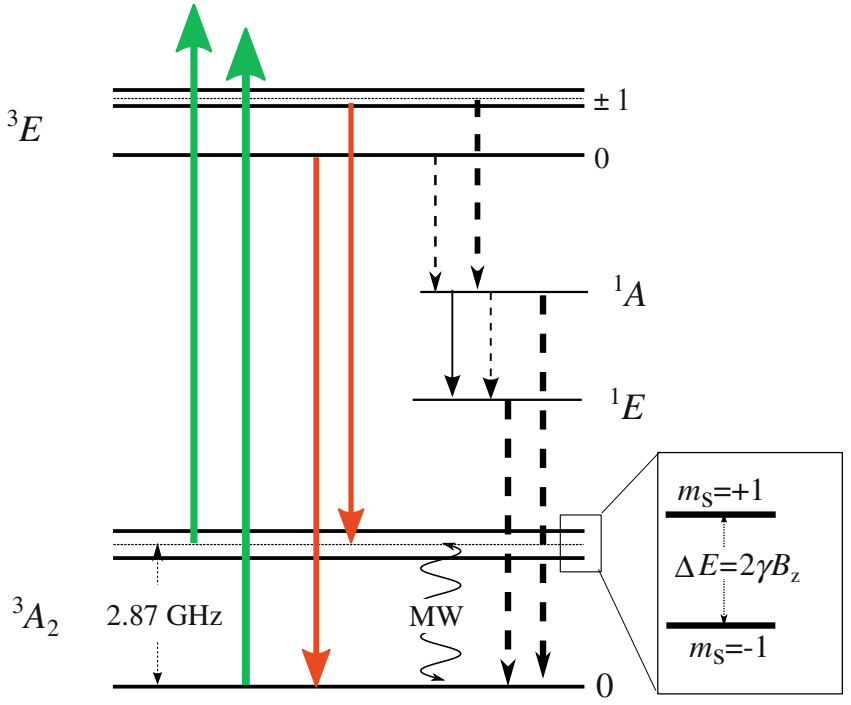

Fig. 1. Level diagram of nitrogen vacancy centres. Radiative transitions are denoted by solid vertical lines, whereas transitions that are likely nonradiative are denoted by dotted lines. The parameter $\gamma=2.8 \mathrm{MHz} / \mathrm{G}$.

degenerate. The two outer peaks correspond to the NV axes that are aligned with the magnetic field. One peak corresponds to $m_{S}=+1$, and the other corresponds to $m_{S}=-1$. The two inner, larger peaks correspond to the three NV centre orientations that make an angle with the magnetic field axis, such that the projection of the magnetic field on the the NV axes is equal. These peaks have a larger magnitude because they correspond to more possible NV centre orientations. Their displacement from the centre is smaller because the projection of the magnetic field onto their NV axis is smaller. In the other case (Fig. 2b), the magnetic field direction forms the same angle of $109.5^{\circ} / 2$ with all $\mathrm{NV}$ alignment directions, and so the two peaks on each side of $2.87 \mathrm{GHz}$ (the zero-field peak) merge into one. These properties can be exploited in many ways using a wide range of microwave pulse sequences developed for nuclear magnetic resonance experiments. However, as one can see from Figure 2, the simplest approach to measuring constant-in-time or slowly changing fields is based on ODMR signals, and this technique is also usually applied in experiments to image magnetic fields.

The first applications of NV centres in diamond to magnetic field measurements were reported in 2008 [2,3], and since then sensitivities have been increased greatly and the number of applications has grown. The first images of magnetic field distributions produced by microscopic current distributions were obtained in 2011 [6]. Since then images have been obtained of magnetic particles as small as $20 \mathrm{~nm}$ [7] and of magnetosomes inside magneto-tactic bacteria [8]. However, these measurements indicated a magnetic moment 10 times smaller than measured by methods based on the motion of these bacteria in magnetic fields [10]. Therefore, it is of great interest to replicate such measurements with particles of known (a)

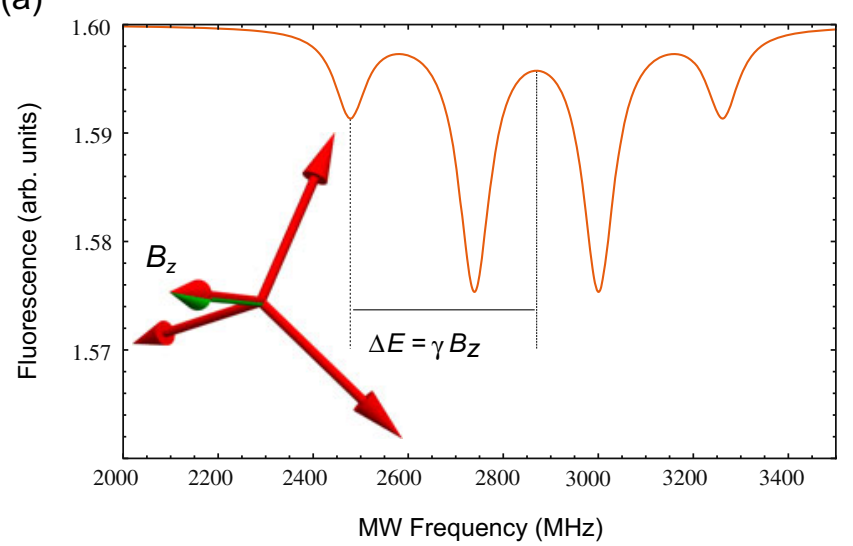

(b)

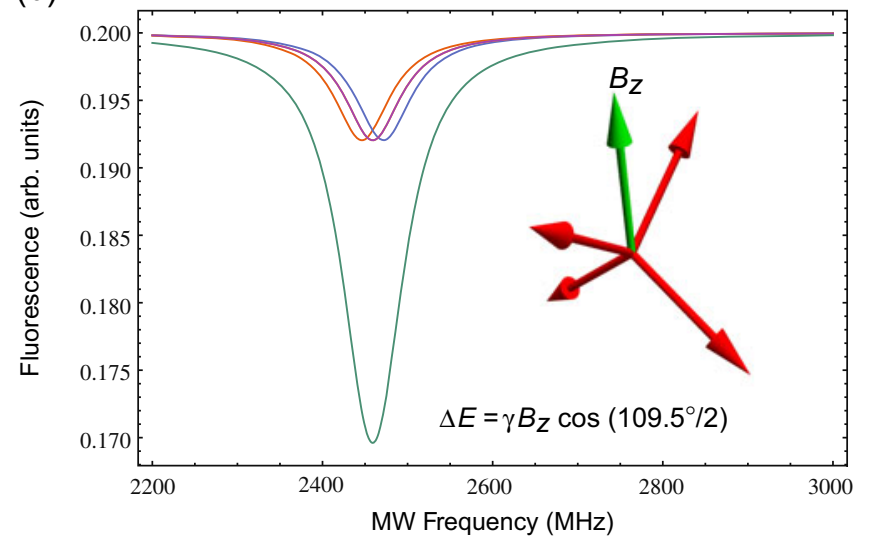

Fig. 2. ODMR (Fluorescence vs. MW frequency) signal for two different configurations of the magnetic field: (a) the magnetic field $B_{z}$ (green arrow) is directed along the $\{111\}$ crystal axis parallel to one of the NV alignments (red arrows); (b) the magnetic field is directed along the $\{100\}$ crystal axis and makes the same angle with all four possible NV alignments. We used the latter configuration in this work.

magnetic moment to see if further insights could be gained into the technique.

The goal of the present work was to obtain images of magnetic field distributions for particles whose magnetic moment have been measured by other means and to compare these measurements to the values obtained from the magnetic field images. In this way, the particular conditions of the measurement can be more carefully evaluated. Then, the determination of unknown magnetic field distributions will become more reliable.

\section{Experiment}

A high-pressure, high-temperature, $\{100\}$ oriented Type 1 b single crystal diamond $(3 \mathrm{~mm} \times 3 \mathrm{~mm} \times 0.1 \mathrm{~mm})$ with a nitrogen content around 100-200 ppm from Element 6 was irradiated with nitrogen ions at three separate energies: $10 \mathrm{keV}, 35 \mathrm{keV}$ and $60 \mathrm{keV}$ and then annealed at high temperature so that the vacancies created by the ions during implantation could migrate to nitrogen defects and form NV centres. According to the software package 
SRIM [12], these vacancies should be distributed between the diamond's surface up to a depth of around $200 \mathrm{~nm}$ (see Fig. 3). However, the extent of defect migration during annealing was not precisely known. Based on measurements of fluorescence as a function of depth, it has been estimated that an initial distribution of vacancies up to a depth of $100 \mathrm{~nm}$ can lead to an NV distribution with a depth of up to $200 \mathrm{~nm} \mathrm{[13].}$

Streptavidin-coated $4 \mu \mathrm{m}$ and $2 \mu \mathrm{m}$ diameter ferromagnetic nanoparticles (Spherotech SVFM-40 and SVFM-20) and $500 \mathrm{~nm}$ diameter superparamagnetic particles (Ademtech MasterBeads Streptavidin) were characterised using a vibrating sample magnetometer to obtain bulk hysteresis curves. The ferromagnetic particles were delivered in a suspension of phosphate buffer $(\mathrm{pH}=7.4)$ with $0.02 \%$ sodium azide. The suspension was diluted 50:1 with distilled water. Then a $10 \mu \mathrm{L}$ droplet was applied to the irradiated surface of the diamond and allowed to dry at room temperature in air in the presence of a magnetic field that oriented the particles parallel to the surface of the diamond as they dried. A thin wire loop was placed on top of the diamond and connected to SMA connectors through which microwaves could be applied. The superparamagnetic particles were supplied by Ademtech in storage buffer (\#10201) and immobilisation buffer (\#10301). After rinsing the diamond, this suspension was diluted 50:1 with distilled water and applied to the diamond.

Figure 4 shows a schematic diagram of the experimental setup used to image magnetic particles and their magnetic field distributions. The diamond was placed on the sample holder of an inverted microscope (Leica DL-ILM). The diamond and the particles on its surface could be illuminated with a light emitting diode for optical imaging. An objective lens with a numerical aperture of 0.65 and a magnification of $40 \times$ was used. To excite the NV centres, $532 \mathrm{~nm}$ laser radiation from a solid state laser (CrystaLaser GC532-100-SLM) was introduced into the microscope base, which contained a filter cube (Leica N2.1), which had an excitation filter with a band-pass range of $515 \mathrm{~nm}$ to $560 \mathrm{~nm}$, a dichroic mirror with a cutoff wavelength at $580 \mathrm{~nm}$, and a suppression filter with a long-pass cutoff wavelength of $590 \mathrm{~nm}$. In this way, the red fluorescence from the NV centres could be imaged by the microscope and recorded using a CCD camera (Leica DFC310 $\mathrm{FX}$ ).

A magnetic field was applied using a permanent magnet located above the sample with the magnetic field direction perpendicular to the diamond surface. In this configuration, all four possible NV axes make the same angle with the magnetic field, and so the ODMR signal has only two peaks, corresponding to $m_{S}=+1$ and $m_{S}=-1$ (as in Fig. 2b). The magnetic field at the surface of the diamond was around $12 \mathrm{mT}$, and so the two ODMR peaks were centred around $2870 \mathrm{MHz} \pm 336 \mathrm{MHz}$. Microwaves (MW) from a generator (Standford Research Systems SG386) were passed through an MW amplifier (Minicircuits ZHL-16W-43-S+). The MW power before the amplifier was measured to be $-10 \mathrm{dBm}(0.1 \mathrm{~mW})$. The amplifier's gain was around $+45 \mathrm{~dB}$, which would lead to a power at its output around $3 \mathrm{~W}$. The microwave

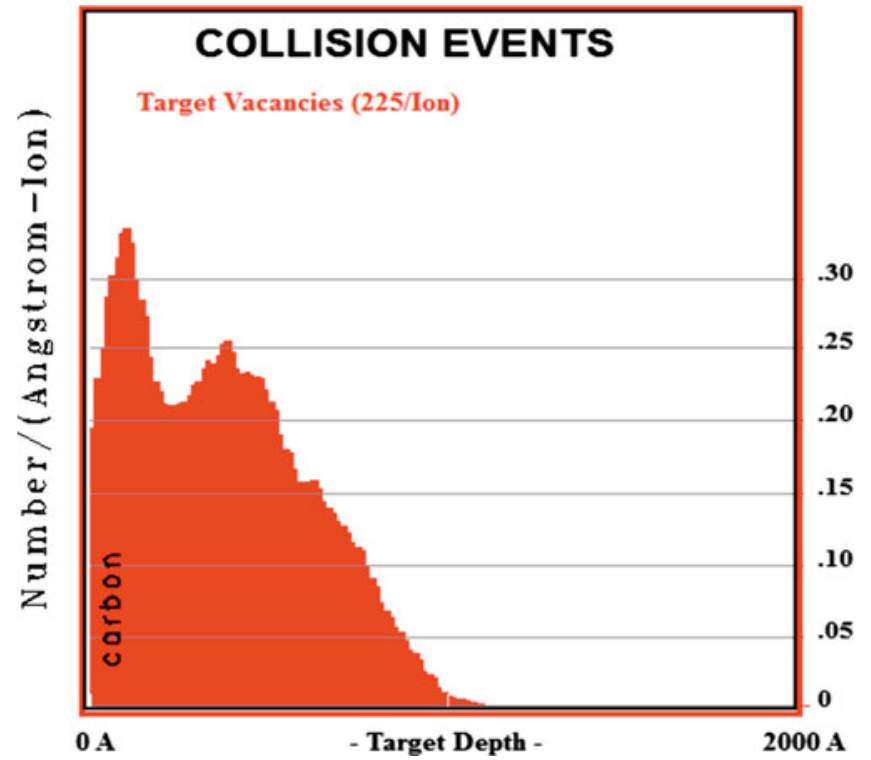

Fig. 3. Relative depth distribution of the ions based on the simulation package SRIM [12]. The vertical axis gives the number of vacancies produced per ion per Ångstrom.

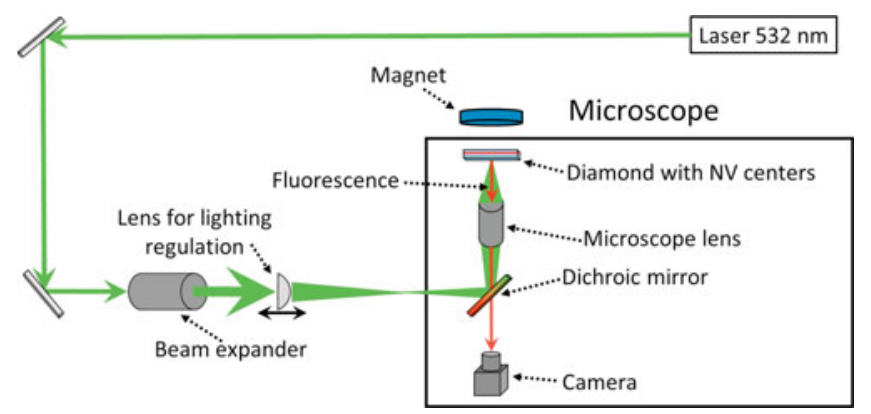

Fig. 4. Schematic diagram of the experiment.

frequency was controlled by custom software, which performed frequency scans to obtain ODMR signals. The scan sequence was as follows. A frequency was selected by the software, and an image $I_{\text {on }}$ was obtained with the MW turned on, and immediately afterwards another image $I_{\text {off }}$ was obtained with the MW turned off. These two images were combined as $I=I_{\mathrm{on}} / I_{\mathrm{off}}$. This procedure was repeated 10 times and the resulting images averaged. Then the sequence was repeated for the next MW frequency. For each frequency an image was saved. The range of the frequency scans was $\pm 2 \mathrm{mT}$ around the ODMR peak of the ambient field in steps of $0.5 \mathrm{MHz}$ for the ferromagnetic particles and $0.2 \mathrm{MHz}$ for the superparamagnetic particles. The scans were repeated 50-100 times, and the results were averaged.

\section{Results and discussion}

The point-spread function (PSF) of the microscope distorts the images for each frequency slightly, which is especially significant for the $500 \mathrm{~nm}$ particles, whose dimension 
The European Physical Journal Applied Physics


Fig. 5. Optical image of the $4 \mu \mathrm{m}$ ferromagnetic particles under white light illumination and pseudo-colour image of the ODMR peak frequency for each pixel.
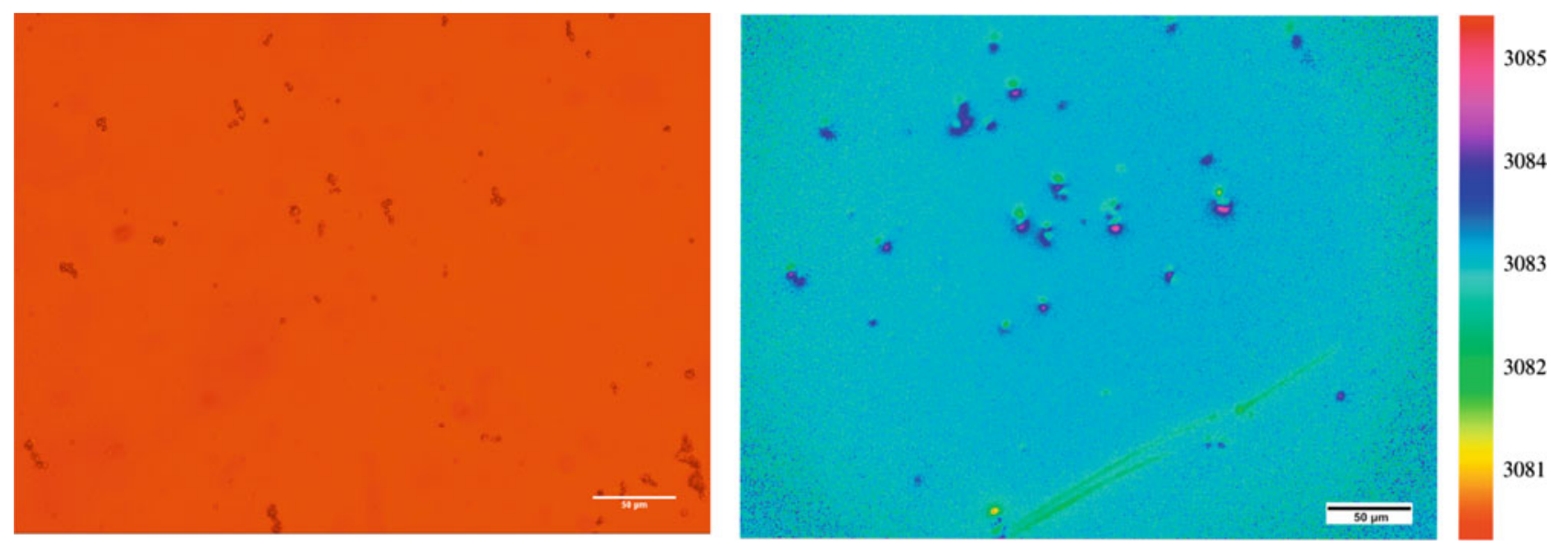

Fig. 6. Optical image of the $2 \mu \mathrm{m}$ ferromagnetic particles under white light illumination and pseudo-colour image giving the ODMR peak frequency for each pixel.
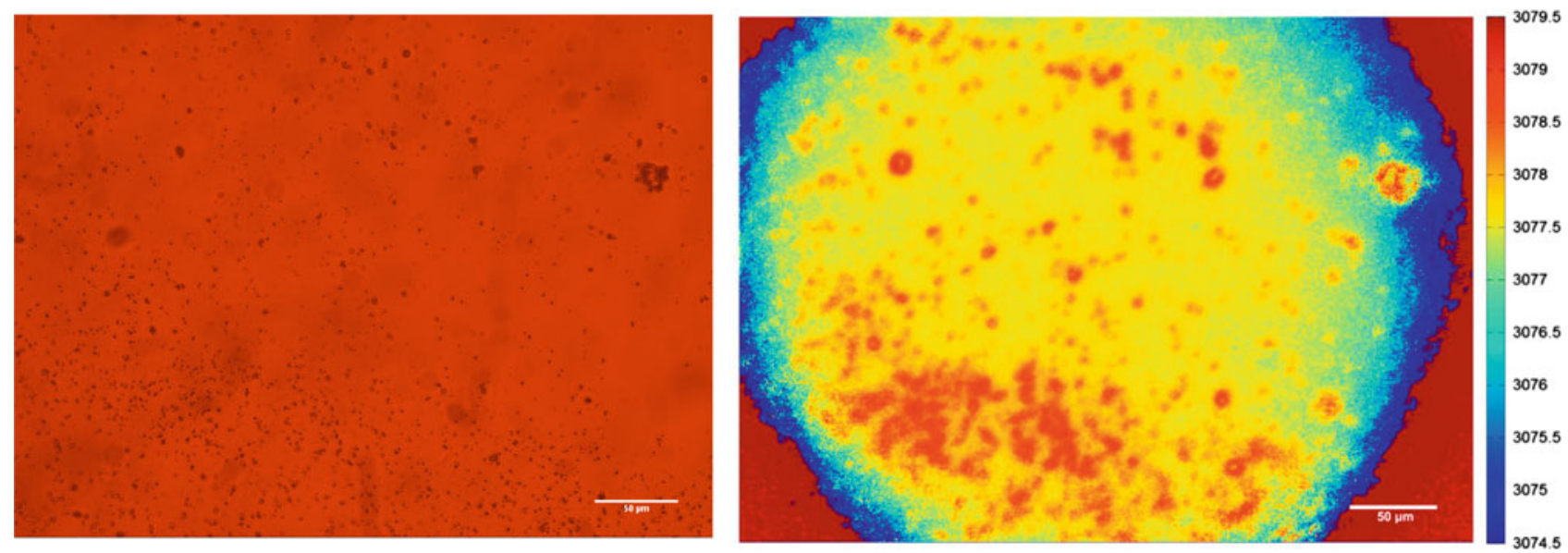

Fig. 7. Optical image of the paramagnetic particles under white light illumination and pseudo-colour image giving the ODMR peak frequency for each pixel. 
(a)

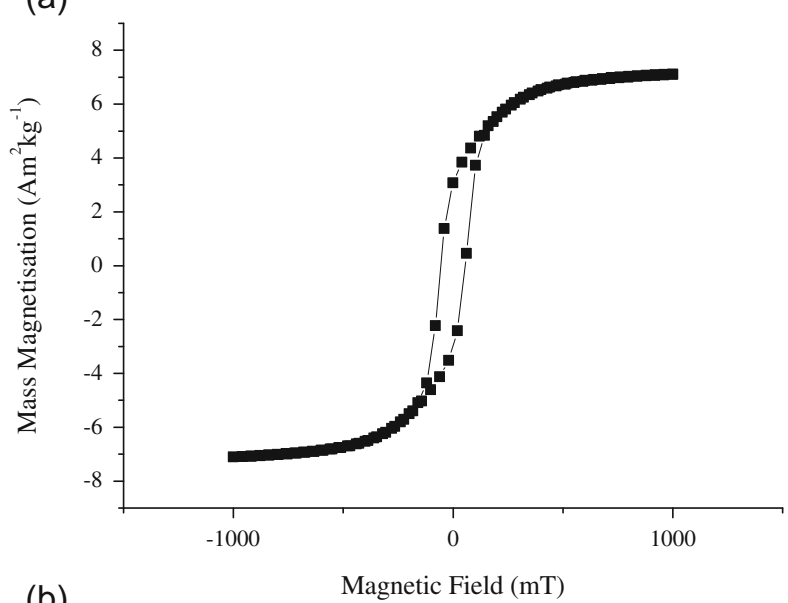

(b)

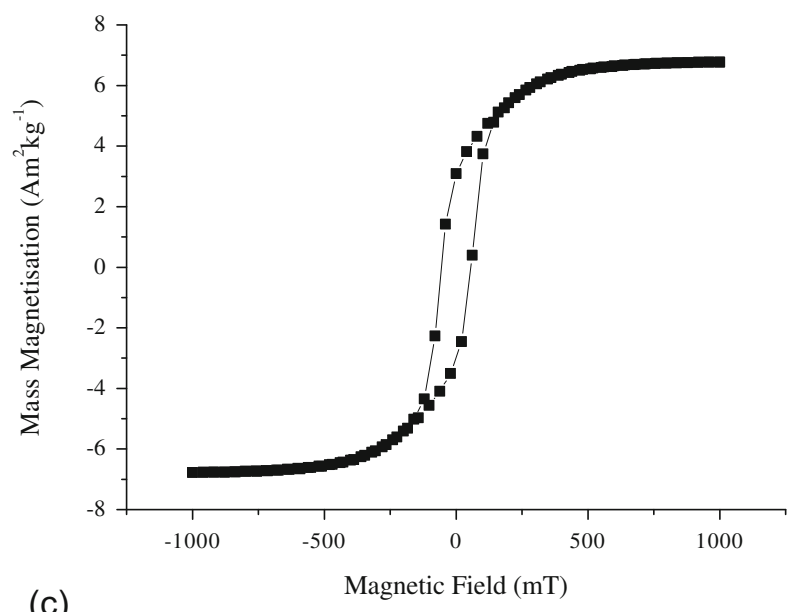

(c)

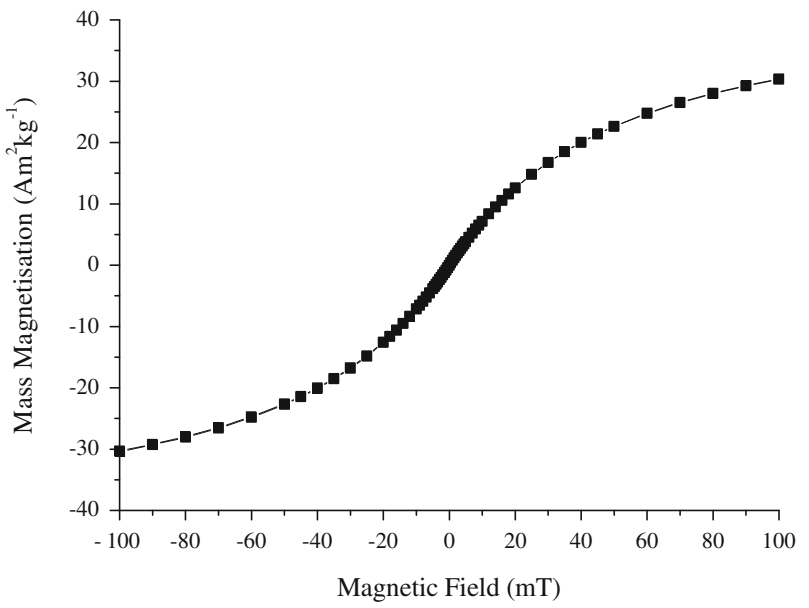

Fig. 8. Measured hysteresis curves of (a) $4 \mu \mathrm{m}$ diameter ferromagnetic particles, (b) $2 \mu \mathrm{m}$ diameter ferromagnetic particles, and (c) $500 \mathrm{~nm}$ diameter superparamagnetic particles. Note different horizontal scales.

is close to the width of the PSF. We used a RichardsonLucy deconvolution with a PSF assumed to be a Gaussian with a standard deviation of $370 \mathrm{~nm}$, which is a good approximation to the central part of an Airy pattern, but computationally less taxing.

Next we searched for the ODMR peaks for each pixel. Since fitting a curve to the ODMR signal for each pixel

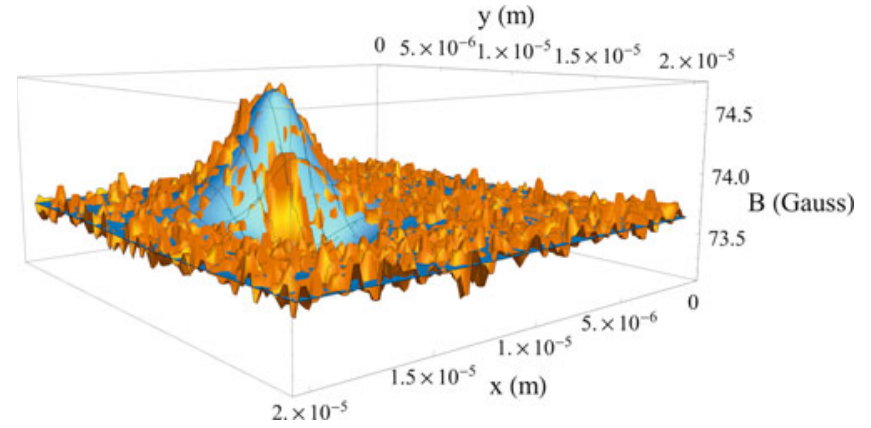

Fig. 9. Measured and best-fit ODMR peak distribution for a single $4 \mu \mathrm{m}$ diameter ferromagnetic particle.

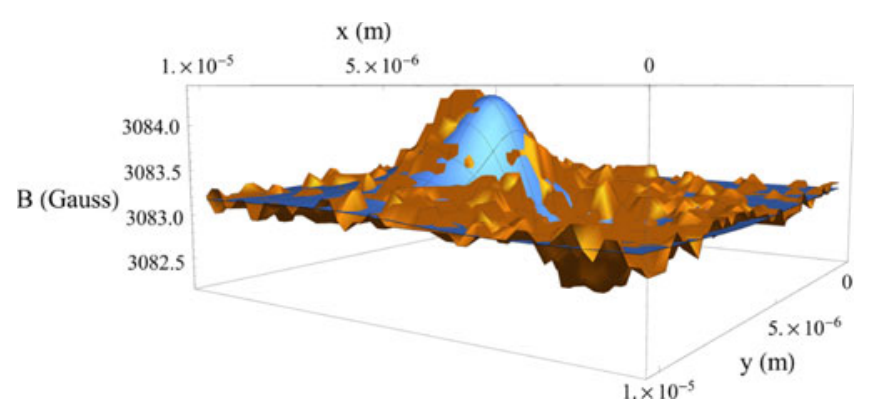

Fig. 10. Measured and best-fit ODMR peak distribution for a single $2 \mu \mathrm{m}$ diameter ferromagnetic particle.

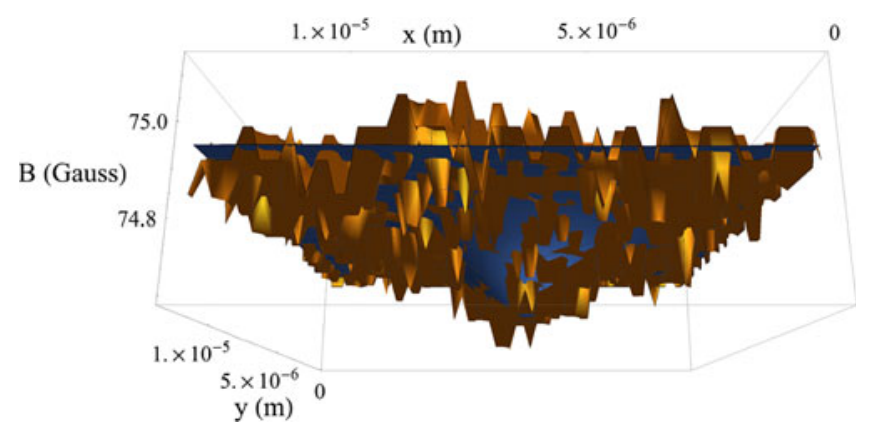

Fig. 11. Measured and best-fit ODMR peak distribution for a single $500 \mathrm{~nm}$ diameter superparamagnetic particle.

would have been time consuming, the ODMR peak was found in the following way. The ODMR signal $f(\omega)$ (see Fig. 2) was transformed to $f^{\prime}(\omega)=-f(\omega)+b$, where $b$ was chosen so that $f^{\prime}(\omega)=0$ for frequencies $\omega$ far from the ODMR peak at $\omega_{0}$ ODMR curve. The peak position was assumed to correspond to the point at which the integral over the entire measurement range from $\omega_{1}$ to $\omega_{2}, \int_{\omega_{1}}^{\omega_{0}} f^{\prime}(\omega) d \omega=\frac{1}{2} \int_{\omega_{1}}^{\omega_{2}} f^{\prime}(\omega) d \omega$, reached half of its maximum value. Then a pseudo-colour image was generated in which the colour of each pixel represents the ODMR peak value obtained for that pixel. Figures 5 and 6 show optical images and the associated pseudo-colour images of ODMR peak values for $4 \mu \mathrm{m}$ and $2 \mu \mathrm{m}$ diameter ferromagnetic particles, respectively. Figure 7 shows optical and pseudo-colour images for the $500 \mathrm{~nm}$ particles.

Fitting a general magnetic field to images containing complex magnetic structures or many particles would 
The European Physical Journal Applied Physics

Table 1. Fitting parameters of the magnetic particles. The parameter $z-r$ represents the height of the particle above the surface of the diamond. The errors given are those resulting from the fit procedure, except in the case of the parameter $z-r$, where the distribution of the radius value $r$ of the spheres is assumed to be on the order of $0.1 \mu \mathrm{m}$.

\begin{tabular}{cccc}
\hline $\begin{array}{c}\text { Particle } \\
\text { type } \\
\text { radius } r(\mu \mathrm{m})\end{array}$ & $\begin{array}{c}\text { Spherotech SVFM-40 } \\
\text { ferromagnetic } \\
2.1\end{array}$ & $\begin{array}{c}\text { Spherotech SVFM-20 } \\
\text { ferromagnetic } \\
1.0\end{array}$ & $\begin{array}{c}\text { Ademtech MasterBead } \\
\text { superparamagnetic }\end{array}$ \\
\hline Expected $|\mathbf{m}|\left(\mathrm{A} \mathrm{m}^{2}\right)$ & $2.3 \times 10^{-13}$ & $2.6 \times 10^{-14}$ & 0.25 \\
\hline Fitted $x(\mu \mathrm{m})$ & $12.62 \pm 0.01$ & $4.99 \pm 0.02$ & $6.3 \times 10^{-15}$ \\
Fitted $y(\mu \mathrm{m})$ & $9.21 \pm 0.01$ & $6.11 \pm 0.02$ & $5.5 \pm 0.1$ \\
Fitted $z-r(\mu \mathrm{m})$ & $1.4 \pm 0.1$ & $1.3 \pm 0.1$ & $3.6 \pm 0.1$ \\
Fitted $m_{x}\left(\mathrm{~A} \mathrm{~m}^{2}\right)$ & $-3.2 \pm 0.3 \times 10^{-15}$ & $-7.7 \pm 0.2 \times 10^{-15}$ & $-2.0 \pm 0.1 \times 10^{-15}$ \\
Fitted $m_{y}\left(\mathrm{~A} \mathrm{~m}^{2}\right)$ & $-6.0 \pm 0.1 \times 10^{-14}$ & $-7.8 \pm 0.2 \times 10^{-16}$ & $1.3 \pm 0.1 \times 10^{-15}$ \\
Fitted $m_{z}\left(\mathrm{~A} \mathrm{~m}^{2}\right)$ & $-5.6 \pm 0.3 \times 10^{-14}$ & $1.8 \pm 0.1 \times 10^{-15}$ & $-1.2 \pm 0.1 \times 10^{-14}$ \\
Fitted $|\mathbf{m}|\left(\mathrm{A} \mathrm{m}^{2}\right)$ & $6.0 \pm 0.1 \times 10^{-14}$ & $-7.9 \pm 0.2 \times 10^{-15}$ & $1.2 \pm 0.1 \times 10^{-14}$ \\
\hline
\end{tabular}

have been a time-consuming and possibly highly underdetermined process. Therefore, a small section of the image was selected, containing, for example, a single magnetic particle. The magnetic field $B_{\text {dip }}$ at position $\mathbf{r}$ of a dipole centred at the origin is given by:

$$
B_{\text {dip }}(\mathbf{r})=\frac{\mu_{0}}{4 \pi}\left(\frac{3 \mathbf{r}(\mathbf{m} \cdot \mathbf{r})}{r^{5}}-\frac{\mathbf{m}}{r^{3}}\right)
$$

where $\mu_{0}$ is the permeability of free space and $\mathbf{m}$ is the magnetic moment.

The magnetic moment of the particles was determined from bulk measurements with a vibrating sample magnetometer (see Fig. 8). From the quoted density of the particles $\left(\rho=1.81 \mathrm{~g} \mathrm{~cm}^{-3}\right.$ in the case of the ferromagnetic particles and $\rho=4.0 \mathrm{~g} \mathrm{~cm}^{-2}$ in the case of the superparamagnetic particles), we determined the magnetic moment of a single ferromagnetic particle as $|\mathbf{m}|=(4 / 3) \pi r^{3} \rho M$, where for the $4 \mu \mathrm{m}$ diameter particles, $r=2.1 \mu \mathrm{m}$ and $M=3.1 \mathrm{~A} \mathrm{~m}^{2} \mathrm{~kg}^{-1}$ was the mass magnetisation determined from Figure 8, whereas for the $2 \mu \mathrm{m}$ diameter particles, $r=1 \mu \mathrm{m}$ and $M=3.3 \mathrm{~A} \mathrm{~m}^{2} \mathrm{~kg}^{-1}$. The results were $2.3 \times 10^{-13} \mathrm{~A} \mathrm{~m}^{2}$ and $2.6 \times 10^{-14} \mathrm{~A} \mathrm{~m}^{2}$ for the $4 \mu \mathrm{m}$ and $2 \mu \mathrm{m}$ diameter ferromagnetic particles, respectively. Similarly $M=8.3 \mathrm{~A} \mathrm{~m}^{2} \mathrm{~kg}^{-1}$ for the superparamagnetic particles at a field of $B=13 \mathrm{mT}$, which gave $2.3 \times 10^{-15} \mathrm{~A} \mathrm{~m}^{2}$ for the $500 \mathrm{~nm}$ diameter particles. We should note that the ferromagnetic particles consisted of a shell of $\mathrm{CrO}_{2}$ particles surrounding a polystyrene core, which implies that there are multiple magnetic domains possible. In the event that the $\mathrm{CrO}_{2}$ particles are uniformly magnetized, the entire sphere can be treated as a single dipole. However, if the different domains have different magnetisations, this assumption no longer holds. The superparamagnetic particles, on the other hand, have an iron oxide core $(>70 \%$ of the volume), and should respond uniformly to an applied magnetic field.

To determine the magnetic moment of the particles from the image, we followed a procedure similar to the one described in reference [8]. We solved the forward problem by computing the magnetic field at the NV layer of the diamond sample. We note that the vacancies were actually distributed through a layer of at least $100 \mathrm{~nm}$, though the vacancies might have migrated in the diamond to a depth of $200 \mathrm{~nm}$ during annealing, based on a comparison with the results Santori et al. [13]. In the case of the $2 \mu \mathrm{m}$ and $4 \mu \mathrm{m}$ diameter particles, the exact distribution of $\mathrm{NV}$ centres in the diamond is not so important as long as the range in which the NV centres may be found is not too large in comparison with the particle diameter. However, for the $500 \mathrm{~nm}$ particles this could be a source for error, or conversely, an opportunity to study the depth distribution of NV centres by solving an inverse problem for magnetic particles of known magnetic moment and distance above the diamond surface. Nevertheless, the best fit for the superparamagnetic particles ended up locating the particles rather distant from the surface of the diamond (possibly due to solidified solute from the buffer), in which case the effects of an NV distribution would be mitigated.

The resultant ODMR image was calculated and compared with the measured ODMR image corrected for the PSF. A nonlinear fitting routine (Mathematica 10.1 [14]) was used to determine the particle's position, distance from the surface of the diamond, magnetic moment, and magnetic moment orientation. The results of the fitted ODMR distributions and measured ODMR distributions are shown in Figures 9-11 and summarised in Table 1. We can see that the fitted magnetic moment of the ferromagnetic particles was about 3-4 times smaller than the value expected based on the vibrating sample magnetometer measurements. The best fit position was about $1.3 \mu \mathrm{m}$ above the surface of the diamond. The particles may have been separated from the diamond surface due to the presence of solute from the buffer solutions. This distance could be estimated based on the known concentration of solutes in the suspension containing the magnetic particles. For the ferromagnetic particles, if we assume that the solute in $10 \mu \mathrm{L}$ was uniformly distributed across the diamond, then we obtain a layer of about $1.2 \mu \mathrm{m}$, of which around $240 \mathrm{~nm}$ correspond to the $0.02 \%$ sodium azide solution and the rest to the $\mathrm{pH}=7.4$ phosphate buffer diluted 50 times. The fits for the ferromagnetic particles gave the same distance between the diamond surface and the particle surface, as they should, since they were suspended in solutions with identical composition. As a result, though the vibrating sample magnetometer and 
the magnetic field imaging gave different results, there was some internal consistency in the case of the ferromagnetic particles. The value of the magnetic moment for the superparamagnetic particles was about four times larger than expected. The exact buffer composition of the superparamagnetic particles was not known.

\section{Conclusion}

We have imaged the magnetic field distributions of particles with known magnetic properties by measuring the ODMR peak of NV centre fluorescence on a pixel by pixel basis. The results of our measurements for two different ferromagnetic particles were 3-4 times lower than expected results based on measurements with a vibrating sample magnetometer and a factor of four larger in the case of the superparamagnetic particle, but the measurements of the ferromagnetic particles showed internal consistency. Obviously, further refinements are needed for the techniques to reach the same result to within measurement error with different methods. Our studies are useful for investigating the conditions of the diamond used for magnetic field imaging, and thus calibrating the ODMR image contrast against magnetic dipole moment. Greater care in avoiding a solute layer when applying the magnetic particles to the diamond surface would allow using this procedure to determine also the distribution of NV centres as a function of distance below the diamond surface by solving an inverse problem for measurements from magnetic particles of different sizes.

The distribution of NV centres in a diamond and the optimisation of different techniques for creating vacancies remains a subject of intense research. NV centres in diamond provide an attractive method for imaging magnetic field distributions in many research problems, such as the study of magnetosomes in magnetotactic bacteria, or the manipulation of cells for medical purposes by introducing magnetic nanoparticles into them.

This work was supported by ERAF Project Nr. 2014/0031/ 2DP/2.1.1.1.0/14/APIA/VIAA/021.

We thank Sean Lourette, Andrey Jarmola, and Guntars Kitenbergs for useful discussions, and M.M. Maiorov for the magnetisation measurements of the samples. Special thanks to Marcis Auzinsh for suggestions and support.

\section{References}

1. F. Jelezko, J. Wrachtrup, Phys. Stat. Sol. A 203, 3207 (2006)

2. G. Balasubramanian, I.Y. Chan, R. Kolesov, M. Al-Hmoud, J. Tisler, C. Shin, C. Kim, A. Wojcik, P.R. Hemmer, A. Krueger, T. Hanke, A. Leitenstorfer, R. Bratschitsch, F. Jelezko, J. Wrachtrup, Nature 455, 648 (2008)

3. J.R. Maze, P.L. Stanwix, J.S. Hodges, S. Hong, J.M. Taylor, P. Cappellaro, L. Jiang, M.V. Gurudev Dutt, E. Togan, A.S. Zibrov, A. Yacoby, R.L. Walsworth, M. Lukin, Nature 455, 644 (2008)

4. L. Rondin, J.-P. Tetienne, T. Hingant, J.-F. Roch, P. Maletinsky, V. Jacques, Rep. Prog. Phys. 77, 056503 (2014)

5. R. Schirhagl, K. Chang, M. Loretz, C.L. Degen, Annu. Rev. Phys. Chem. 65, 85 (2014)

6. L.M. Pham, D. Le Sage, P.L. Stanwix, T.K. Yeung, D. Glenn, A. Trifonov, P. Cappellaro, P.R. Hemmer, M.D. Lukin, H. Park, A. Yacoby, R.L. Walsworth, New J. Phys. 13, 045021 (2011)

7. M. Gould, R.J. Barbour, N. Thomas, H. Arami, K.M. Krishnan, K.-M.C. Fu, Appl. Phys. Lett. 105, 072406 (2014)

8. D. Le Sage, K. Arai, D.R. Glenn, S.J. DeVience, L.M. Pham, L. Rahn-Lee, M.D. Lukin, A. Yacoby, A. Komeili, R.L. Walsworth, Nature 496, 486 (2013)

9. S. Steinert, F. Ziem, L.T. Hall, A. Zappe, M. Schweikert, N. Gotz, A. Aird, G. Balasubramanian, L. Hollenberg, J. Wrachtrup, Nat. Comms. 4, 1607 (2013)

10. R. Nadkarni, S. Barkley, C. Fradin, PLoS One 8, e82064 (2013)

11. K. Erglis, Q. Wen, V. Ose, A. Zeltins, A. Sharipo, P.A. Janmey, A. Cebers, Biophys. J. 93, 1402 (2007)

12. J.F. Ziegler, M.D. Ziegler, J.P. Biersack, Nucl. Instrum. Methods Phys. Res. B 268, 1818 (2010)

13. C. Santori, P.E. Barclay, K.-M.C. Fu, R.G. Beausoleil, Phys. Rev. B 79, 125313 (2009)

14. I. Wolfram Research, Mathematica, Version 10.1 ed. (Wolfram Research, Inc., Champaign, IL, 2015)

Open Access This article is distributed under the terms of the Creative Commons Attribution License http:// creativecommons.org/licenses/by/4.0 which permits unrestricted use, distribution, and reproduction in any medium, provided the original author(s) and source are credited. 